\title{
Active element influence on the motor's torque
}

\author{
Antonina Dolgih*, Vladimir Martemyanov and Valeriy Borikov \\ Tomsk Polytechnic University, Institute of Non-Destructive Testing, 634050, Tomsk, Russia
}

\begin{abstract}
The paper presents the numerical and experimental studies of the influence of the torque motor active element on the motor's torque. The tape active element is a novel type of a motor's stator organization, where the conventional winding is replaced by a tape winding. The force (torque) dependence over the rotor pole position using COMSOL is given; the tape winding resistance and the turns number are defined. The relative motor's characteristics are investigated and the maximum torque over the certain poles pair number is obtained. The application of the proposed active element in brushless DC motor is considered. The results show the possibility of the further synthesis of the torque motor.
\end{abstract}

\section{Introduction}

The advantages of torque motors allow using them in the direct drive development. This is confirmed by the fact that, at present, many companies develop and improve such motors. The history of the torque motors development and the problems of their improvement in details are described in the works of Alexander Mikerov $[1,2]$. Theoretical principles of the widespread brushless permanent magnet motors are given in the majority of known sources, for example [3-5]. Nowadays, in order to improve the torque motors characteristics, both magnetic circuits and windings are being investigated $[6,7]$.

The particular attention is paid to the thermal mode of the motor operation, since a high temperature can destroy the winding and degrade the permanent magnets properties. The increased temperature during the operation is caused by the large current flowing through the windings. The motor overheating reduction can be achieved by its intensive cooling. The fan application connected to the rotor is not rational due to the low rotation speed. Therefore, in some cases, liquid cooling is used. For example, RIB torque motors [8] are slotted, permanent magnet direct drive motors with an internal rotor. The primary part is a fully cast stator with external liquid cooling. On the outer stator surface there are ring canals. TMB motors [9] also contain a canal structure with a cage for water cooling. However, this cooling method is difficult to use when the torque motor represents a part of a system located on the moving objects. In this situation, the torque motor, whose stator active element is a multilayer winding from a conductive tape with side cuts, is preferable [10].

The benefits of this active element include the novel design and technological approach to the motor stator manufacture; the possibility of high current load with a good heat removal; the formation of required functional dependence of the motor's torque over the angular movement.
The active element presents a set of serially connected plates, through which the current flows in the diagonal direction. The current transverse components interact with the magnetic field of the rotor poles and create a torque. The length of each plate $a$ is equal to the pole division $\tau$ of the motor magnetic circuit. Diagonal character of current, flowing on each plate, is provided by the side cuts, located from the each tape side with a pole division pitch [11].

The purpose of this work is to show the influence of the active element characteristics on the motor's torque.

\section{The active element characteristics}

\subsection{Torque dependence}

The character of current flowing through the active element is distributed. The force $F$ acts on the rotor pole and depends on the flux density $B$, the tape thickness $\Delta$ and the double integral from the distributed current density [12]:

$$
F=B \cdot \Delta \cdot \iint j(x, y) d x d y .
$$

This force can be determined numerically using COMSOL software $[11,12]$.

Generally, the force dependence $F(x)$ over the relative position of plate and rotor pole has the form shown in Figure 1. Accordingly, the torque dependence $T$ over the rotor angular position $\alpha$ will also be displayed in the same way.

The character of the resulting dependence $M(\alpha)$ in many practical cases is unacceptable. For the required dependence, it is proposed to apply the additional side cuts along with the main ones. An example of this method is shown in Figure 2.

\footnotetext{
* Corresponding author: ivanovatonya@tpu.ru
} 


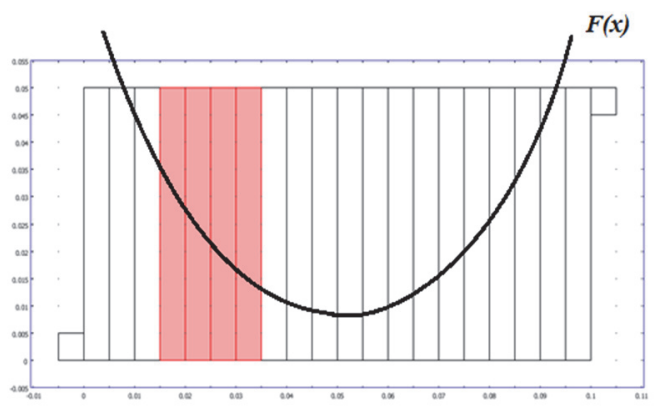

Fig. 1. The force dependence over the rotor pole position.

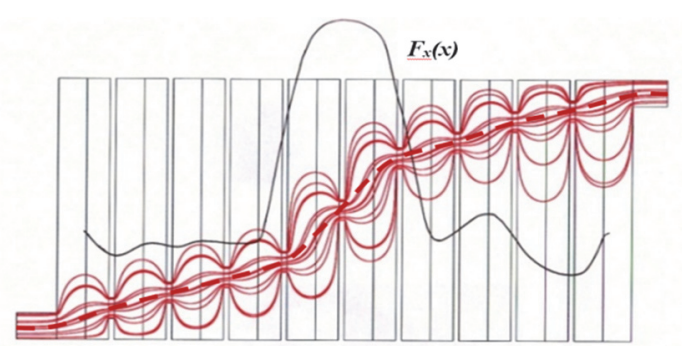

Fig. 2. The required force dependence using the additional side cuts.

The particular interest represents "the triangular" torque dependence over the angular position. This dependence can be realized with only two additional cuts on each plate (Fig. 3a). Figure $3 \mathrm{~b}$ shows the dependence of the double integral $D(x)$ over the plate length. The double integral is taken from the expression (1).

\subsection{Plate resistance}

During the numerical simulation of the proposed active element, it was found that the resistance of a single plate depends on the ratio of its length $a$ and width $b$. Varying this ratio $\beta=a / b$, a certain minimum resistance value is observed (Fig. 4). The parameter $\beta_{\mathrm{e}}=b_{e} / b$ is the relative electrode width, where $b_{e}$ is the tape section size, remaining after the cut.

Besides, the plate resistance is defined experimentally using thin aluminum tape with the following geometrical parameters: $b=0,05 \mathrm{~m} ; \beta_{\mathrm{e}}=0,2$; $\Delta=0,0007 \mathrm{~m}$. The results are shown in Figure 5 .

\subsection{Winding turns number}

Let us suppose that the number of winding turns $N$ is small, and the plates geometry in all layers is almost similar. Then the each plate resistance $R$ will be permanent. Also, suppose that the magnetic flux density is uniform.

As shown earlier, the force acting on one plate is defined as:

$$
F=\Delta \cdot B \cdot D\left(I_{0}, x_{c}\right)
$$

Further, for simplicity, the double integral $D\left(I_{0}, x_{c}\right)$ over the area, bounded by the magnetic flux density action and with the center determined by the coordinate $x_{c}$, will be denoted by $D$. The value of this integral is determined by passing through the plate the current $I_{0}$.

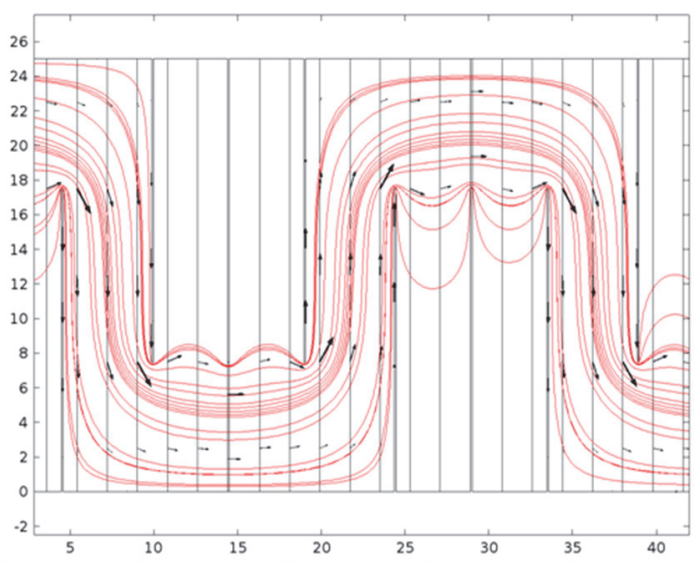

a)

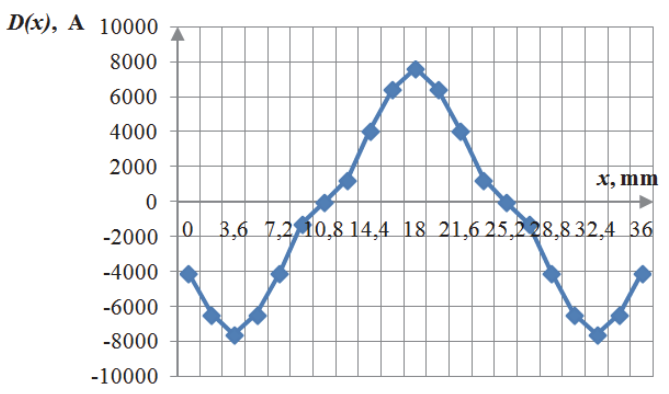

b)

Fig. 3. "The triangular" torque dependence.

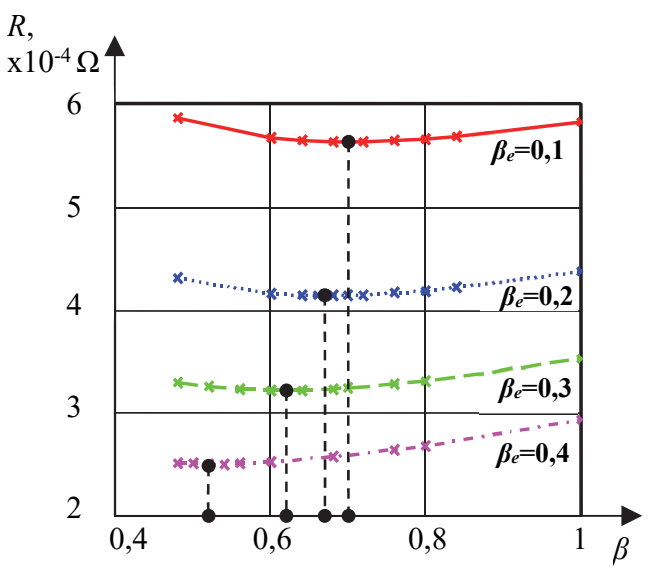

Fig. 4. The dependence of the plate resistance over the ratio of its length and width.

The current flowing through the tape winding depends on the source voltage $U$, the plate active resistance $R$, the number of poles pairs $p$, the number of winding turns $N$ :

$$
I=\frac{U}{2 p \cdot N \cdot R}
$$

Since the force is determined by the double integral $D$, which depends linearly on the current, let us write the expression for the force in the following form: 


$$
F=\Delta \cdot B \cdot \frac{U}{2 p \cdot N \cdot R \cdot I_{0}} \cdot D
$$

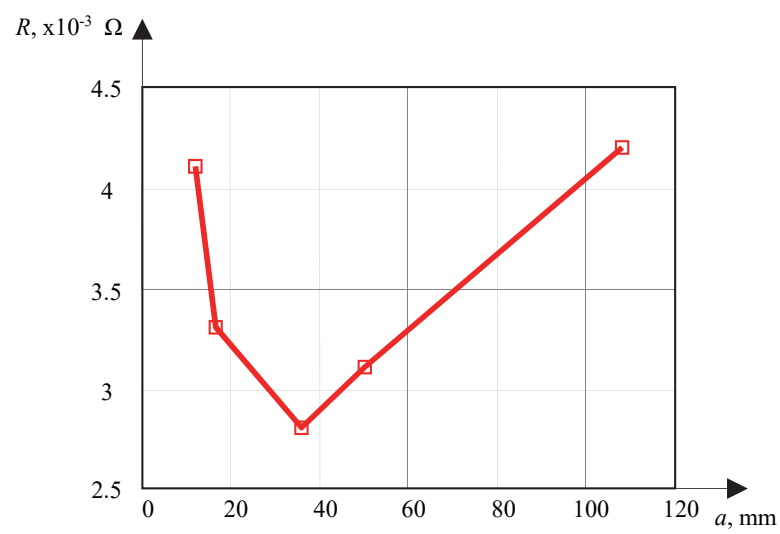

Fig. 5. The experimental dependence of the plate resistance over the ratio of its length and width.

The motor's torque is equal to the sum of the torques, created by each plate of all winding layers. The winding average radius is defined as:

$$
r_{a v}=r_{0}+\frac{N \cdot \Delta}{2}
$$

where $r_{0}$ is the tubular base radius on which the tape is wound.

In this case, the torque is determined as follows:

$$
T=2 p \cdot N \cdot F \cdot r_{a v}=\Delta \cdot B \cdot \frac{U}{R \cdot I_{0}} \cdot D \cdot\left(r_{0}+\frac{N \cdot \Delta}{2}\right) \text {.(6) }
$$

Let us estimate the influence of the number of winding turns on the developed torque value. On the one hand, the number of plates increasing should lead to the torque growth. On the other hand, the total tape winding resistance will increase by the same amount, and consequently the current will decrease. In this case, the number of winding turns does not affect the torque value.

The torque change due to the average winding radius increase is insignificant. But at the same time, a smaller number of winding turns will decrease magnetic circuit air gap and lead to the growth of the flux density $B$. When defining the minimum value of $N$, it is necessary to proceed from the possibilities of both the power source and the winding heat resistance. The number of turns must be minimal and is determined by a permitted current:

$$
N \geq \frac{U}{2 p \cdot R \cdot I_{\text {perm }}}
$$

\subsection{Relative characteristics}

Let us introduce the concept of the relative torque to determine the number of magnetic circuit poles pairs and, correspondingly, the winding parameters at which the maximum torque is observed.

$$
k_{M}=\frac{M_{i}}{M_{1}}=\frac{B_{i}}{B_{1}} \cdot \frac{R_{1}}{R_{i}} \cdot \frac{D_{i}}{D_{1}} .
$$

In this case, $M_{1}, B_{1}, R_{l}, D_{1}$ characterize the motor with one poles pair $p=1 ; M_{i}, B_{i}, R_{i}, D_{i}$ characterize the motor with poles pairs number $p=i$.

Firstly, we will only take into account the parameters characterizing the winding. There are the plates resistance and the double integrals of the distributed currents. For their determination the winding numerical models with the average radius range $r_{a v}=0,025 \div 0,075 \mathrm{~m}$ are created in the COMSOL software. The tape width $b$ $=0,05 \mathrm{~m}$ and $0,025 \mathrm{~m}$; the tape thickness $\Delta=10^{-4} \mathrm{~m}$; the tape material is a copper.

The coefficient of pole overlap is $\alpha_{p}=0,5$. The pole center position is at the point $x_{c}=0,25 \cdot a_{i}$, where the plate length $a$ is equal to the pole division $(a=\tau)$. The graphs of the resistances ratio $R_{l} / R_{i}$ over the pole pairs are shown in Figure 6 . The graphs correspond to the case $r_{a v}=0,027 \mathrm{~m}$ and $b=0,025 \mathrm{~m}$.

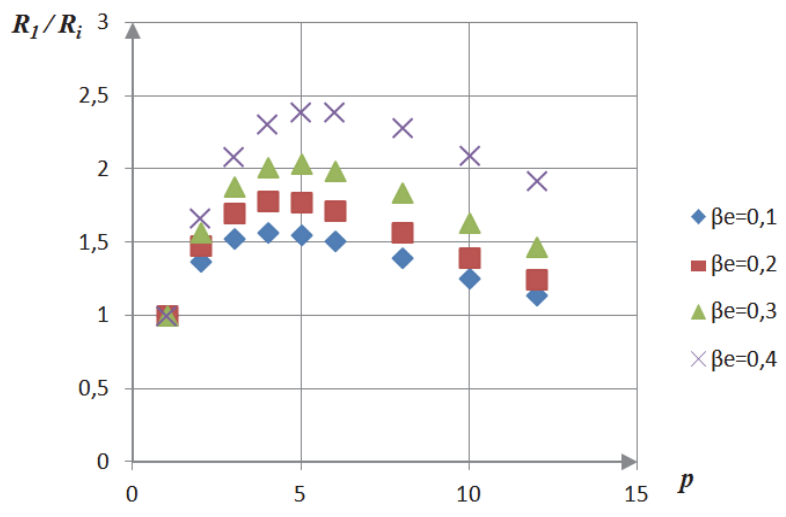

Fig. 6. The character of relative resistances changing.

For other considered variants of tape winding, the characters of relative resistances changing are preserved. Their maximum values are in the range of the poles pairs number $p=4 \div 6$. Figure 7 shows the dependencies of the calculated double integrals values for the tape winding with the parameters given above.

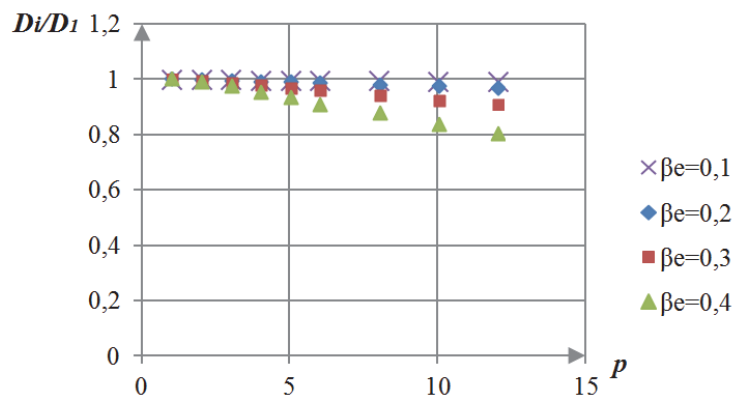

Fig. 7. Relative double integral values changing.

As seen from the graphs (Fig. 7), the double integral value $D$ varies insignificantly with the change in the number of pole pairs, especially for small $\beta_{\mathrm{e}}$. Therefore, we can conclude that the main effect on the motor's 
torque growth exerts the active resistance of the winding plates.

Since the aim of this work is not to define the magnetic circuit optimum parameters, a simple parametric model of the motor was constructed in T-flex CAD with the possibility of changing the poles pairs number and the air gap size (Fig. 8).

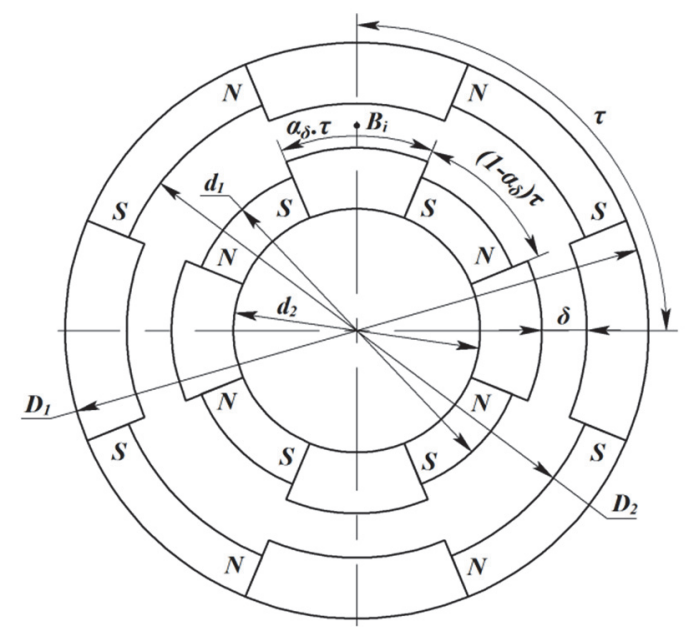

Fig. 8. The model of torque motor magnetic circuit.

Figure 9 shows the flux density changing in the air gap of the investigated magnetic circuit. The air gap $\delta$ varied from 3 to $7 \mathrm{~mm}$.

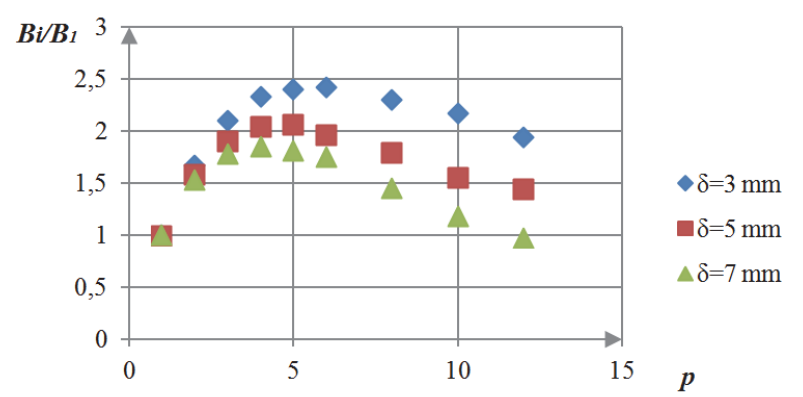

Fig. 9. The flux density changing in the air gap over the different poles pairs number.

Analyzing the obtained results, the maximum torque for the tape winding model of the selected geometric parameters will be observed with the poles pairs number in the range from $p=4$ to $p=6$. The relative torque dependence $k_{T}(p)$ is shown in Figure 10.

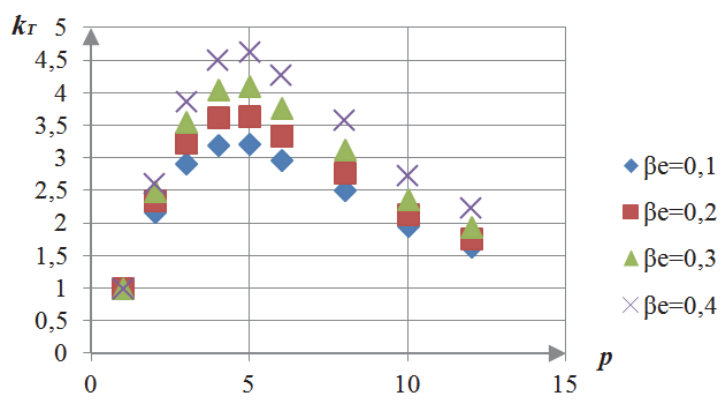

Fig. 10. The relative torque over the different poles pairs number.
The power consumed by the motor is determined by the expression:

$$
P=\frac{U^{2}}{2 p \cdot R \cdot N} .
$$

Similarly to the previous calculations, let us introduce a concept of the relative power consumption:

$$
k_{p}=\frac{P_{i}}{P_{1}}=\frac{R_{1}}{p \cdot R_{i}}=\frac{1}{p} \cdot\left(\frac{R_{1}}{R_{i}}\right) .
$$

The results of calculations for tape winding with the given earlier parameters are shown by the graphs in Figure 11.

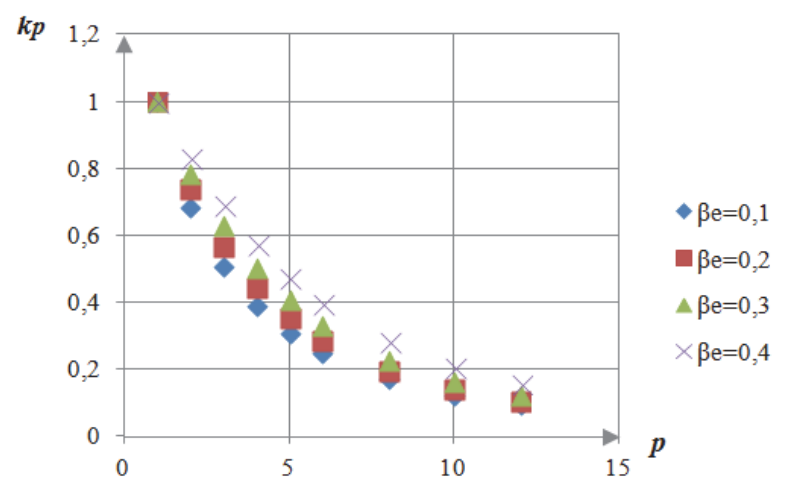

Fig. 11. The relative power consumption over the different poles pairs number.

In practical work, the static Q-factor [13], as the torque motor characteristic, is of interest

$$
Q=\frac{M}{m \cdot P^{0,5}} .
$$

The relative static Q-factor $k_{Q}(p)$ was estimated, taking into account the assumption that the motor mass $m$ increases insignificantly with the increase in the number of poles pairs. The results of the calculations are reflected by the graphs in Figure 12.

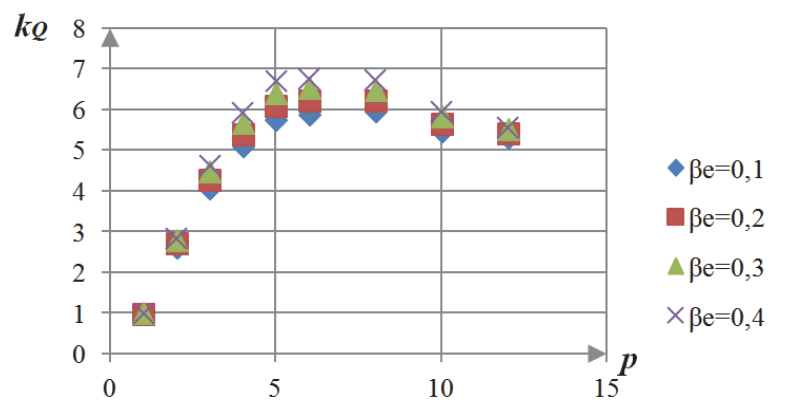

Fig. 12. The relative static Q-factor.

The numerical simulation results show that the torque motor optimum parameters will be observed with the poles pairs numbers from $p=4$ to $p=6$, with the given geometric parameters of the tape winding. 


\section{Results and discussion}

As a result of this work, the force (torque) dependence and the variants of the required torque dependence implementation are shown. The resistance of the tape winding single plate has a minimum value at the certain ratio of the plate length and width. The number of winding turns does not influence the motor's torque. The analysis of the relative motor's characteristics is carried out. The maximum torque is observed at a certain number of poles pairs. The obtained results allow making the synthesis of the proposed torque motor.

Also, it is important to consider the application of the proposed active element in electromechanical devices. The two-phase brushless DC (BLDC) motor can be realized using two tape windings. The cuts of the second tape winding should be shifted relative to the first tape winding by half of the pole division [14].

In this variant, the torque combined characteristic will not have zero points. If the torque dependencies of both windings are identical and represent a combination of isosceles triangles, the resulting motor's torque will be unchanged for all rotor positions. Ideally, the torque ripple will be absent (Fig. 13).

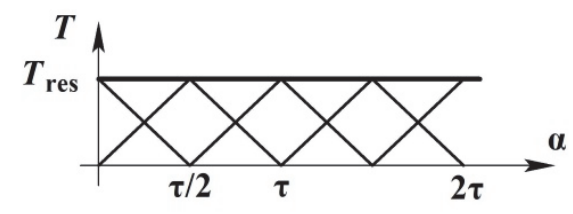

Fig. 13. The two-phase BLDC motor torque.

The second tape winding is coaxial with the first and is under the influence of the same rotor magnetic flux. There are two variants of the second tape winding location: it can be located side by side in the axial direction, performed during simultaneous winding of the two tapes; or it can be placed concentrically, covering the first winding. The design of such motor is shown in Figure 14.

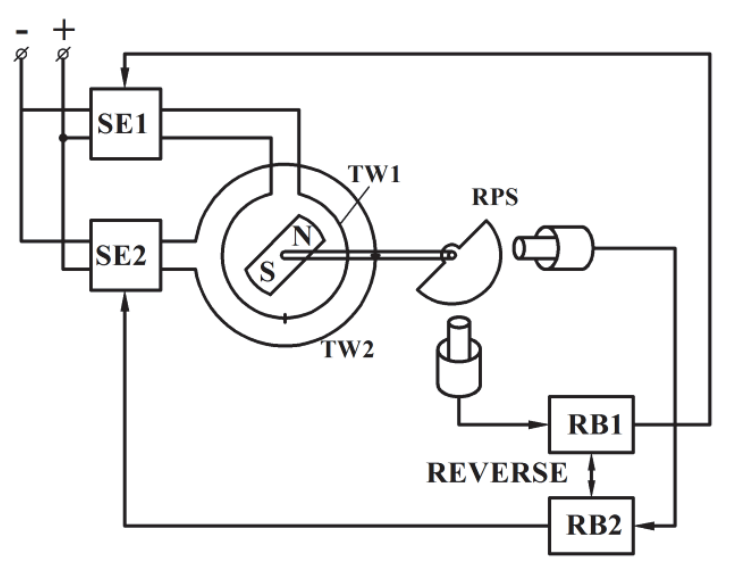

Fig. 14. The BLDC motor design.

The following symbols are used: TW - the tape winding, SE - the switch element, RB - the reverse block, RPS - the rotor position sensor. RPS can be implemented on the inductive sensor and its rotor is made as a disk of ferromagnetic material of the appropriate shape. RPS switches the signal between the tape windings. If it is necessary to change the direction of rotation, as well as the sign of the torque, the tape winding current should flow in an opposite direction. This can be done by the reverse block of the BR, where the signal from RPS is reversed.

\section{Conclusion}

The results of the work show the influence of the characteristics of the proposed active element on the motor's torque and allow continuing the research. At present time, the numerical simulations of torque pulsations with the different current values flowing through the set of two tape windings are being realized.

\section{References}

1. G. Dubrovskiy, A. Mikerov, V. Dzhankhotov, J. Pyrhonen EPE-ECCE Europe, 6910754 (2014)

2. A. G. Mikerov, J.J. Pyrhönen, J.J. Vauterin EUROCON, 4400568 (2007)

3. D. Hanselman, Brushless Permanent Magnet Motor Design. Second Edition. (Magna Physics Publishing, United States of America, 2006)

4. A. Hughes B. Drury, Electric Motors and Drives. Fundamentals, Types and Applications. 4th Edition. (Elsevier-Newnes, Oxford, 2013)

5. P. Madaan, Brushless DC Motors. Part 1. Construction and Operating Principles. (Cypress Semiconductor, India, 2013)

6. Z.Q. Zhu and D. Howe, IEEE Transactions on Energy Conversion 15(407) (2000)

7. L. Zhu, S.Z. Jiang, Z.Q. Zhu, C.C. Chan, IEEE Transactions on Magnetics 45(4), 2023 (2009)

8. INA - Drives \& Mechatronics GmbH \& Co. KG, IDAM Direct Drives (2014)

9. ETEL, Torque Motors. (2017)

10. V. Martemjanov, A. Dolgih (Ivanova), RF2441310, (2012)

11. V. Martemjanov, A. Dolgih (Ivanova), SIBCON, 6693632 (2013)

12. A. Dolgih, V. Martemyanov, MATEC Web of Conf. 48, 01004 (2016)

13. A.V. Demagin, Electric machines for direct drive of instrument systems. (Scientific-Production. Association "Azimut", Saint-Petersburg, 1991) (In Russian)

14. V. Martemjanov, A. Dolgih (Ivanova), RF2454776, (2012) 